\title{
Machine learning prediction models based on hub genes related to immune phenotypes in muscle-invasive bladder cancer treated with immune checkpoint blockade
}

\section{Peiheng Li ( $\square$ lipeiheng@pumc.edu.cn )}

Peking Union Medical College Hospital https://orcid.org/0000-0002-5642-6778

\section{Zhi-Xin Chen}

Peking Union Medical College Graduate School

\section{Dong Wang}

Peking Union Medical College Hospital

\section{Zhi Zheng}

Peking Union Medical College Graduate School

\section{Zhi-Gang Ji}

Peking Union Medical College Hospital

\section{Primary research}

Keywords: Muscle-invasive bladder cancer (MIBC), Immune checkpoint blockade (ICB), Immune microenvironment, Metabolism, Multi-omics analysis, Weighted Gene Co-expression Network Analyses (WGCNA), Classification and regression tree (CART)

Posted Date: April 21st, 2021

DOI: https://doi.org/10.21203/rs.3.rs-63592/v3

License: (c) (i) This work is licensed under a Creative Commons Attribution 4.0 International License. Read Full License 


\section{Abstract \\ Background}

Bladder cancer is one of the most frequent cancers in the world. Muscle-invasive bladder cancer (MIBC) is the aggressive subtype with higher morbidity and mortality. Immune check point blockade (ICB) therapy has shown its potential for treating MIBC, but is limited due to the lack of predictive biomarkers.

\section{Methods}

1601 MIBC transcriptomic profiles were obtained from 10 datasets. Unsupervised clustering of immune phenotypes in MIBC was performed based on immune-related signature genes selected by us. We analyzed the characteristics including microenvironments, metabolic pathways, and survival rates in different phenotypes. Multi-omics analysis and WGCNA plus protein-protein interaction (PPI) analysis were performed to identify hub genes of differentially expressed genes (DEGs) distinguishing phenotypes related to prognosis. The hub DEGs were further validated by real-time quantitative PCR (qPCR). A model was established and CART was employed to predict the responses of patients treated with ICB.

\section{Results}

Of various immune phenotypes, cluster $3 \mathrm{C}$ was the most "inflamed" subcluster with the best prognosis, while cluster $1 \mathrm{~A}$ was associated with "non-inflammation" and worst prognosis. There was no intersection of hub DEGs selected by WGCNA plus PPI analysis and multi-omics analysis. WGCNA plus PPI analysis identified 5 hub genes related to the survival rate of patients, IFNG, CXCR6, IL2RB, LCK, and PSMB10. Real-time qPCR results indicated that the expression levels of 5 hub genes were significantly lower in tumors. The 5 hub genes were further utilized for prognostic score model and decision tree analysis. The areas under the curve (AUC) of the ROC curves predicting 5-year endpoint generated from the risk-based prediction model were 0.652 . The mean accuracy, sensitivity, and specificity of CART for predicting stable disease/progressive disease were $70.1 \%, 70.0 \%$ and $71.7 \%$.

\section{Conclusions}

The 5 hub genes and generated models showed the potential for predicting the prognosis for patients receiving ICB therapy. The molecular mechanisms regulating the expression of the hub genes require further studies in the future.

\section{Introduction}

Bladder cancer was the tenth most frequent cancer worldwide in both sexes combined, resulting in an estimated 549000 new cases and 200000 deaths worldwide in 2018[1]. Most bladder cancers are urothelial carcinomas, which can be divided into non-muscle-invasive bladder cancer (NMIBC) and muscle-invasive bladder cancer (MIBC) according to their distinct prognosis. Compared with NMIBC, MIBC was more aggressive with higher morbidity and mortality, with poor 5-year survival of $40-60 \%[2]$. Treatments for patients with non-metastatic MIBC included cystectomy, extended lymph node dissection, and perioperative chemotherapy. For patients with metastatic MIBC, the response rate of first-line treatment, cisplatin-based chemotherapy, was only 46\%-49\%[2]. Immune checkpoint blockade (ICB) therapy targeting programmed death ligand-1 (PDL1)/PD-1 has shown its potential for MIBC patients who are insensitive to chemotherapy. In 2017, FDA approved 2 ICBs, atezolizumab (a PD-L1 inhibitor) and pembrolizumab (a PD-1 inhibitor), for first-line treatment of cisplatin-ineligible patients[3]. However, the response rate of ICB therapy is also limited due to the tumor's intrinsic heterogeneity and the lack of predictive biomarkers. 
Gene expression profiling has been used to identify molecular heterogeneity in bladder cancer, which could be used to predict responses to different therapies. There was a consensus that bladder cancer can be divided into basal and luminal subtypes, displaying different responses to chemotherapy[4]. A recent study defined a molecular classification of 6 subtypes,

stratifying patients for prognosis and response to treatment[5]. These studies focus mainly on malignant urothelial cells. The prediction of response to immunotherapy is complicated because tumor microenvironment is composed of immune cells and stromal cells, in addition to malignant cells. A recent review proposed that the cancer immunogram involving infiltrated cells and immune related signaling has shown the potential for personalized immunotherapy in bladder cancer[6]. Immunerelated genes play important roles in constructing tumor microenvironment and responding to ICB therapy.

The major objective of our study was to construct prediction models to distinguish immune phenotypes in MIBC and improve the prediction of response to ICB therapy. We established a novel molecular classifier based on immune-related genes selected by us. Different immune phenotypes of MIBC were classified by unsupervised clustering. We then explored the microenvironments including infiltrated immune cells and stromal cells to evaluate the correlated immune signaling and survival rate in each phenotype. The difference of metabolic pathways among various immune phenotypes was also analyzed as the concept of immunometabolism has become widespread[7, 8]. Weighted gene co-expression network analysis (WGCNA) plus PPI is a common tool to unravel the complex relationships among genes and help screen hub differentially expressed genes (DEGs) to distinguish different immune phenotypes of MIBC[9]. Genetic and epigenetic alterations were also related to tumor microenvironment as somatic copy-number alterations were associated with different tumor immune phenotypes[10]. We applied multi-omics data analysis involving somatic mutation, copy-number variation, and DNA methylation analysis to study the establishment of different tumor microenvironments, also trying to highlight molecular principles of DEGs in corresponding phenotypes. The expression levels of hub genes of bladder cancer samples were further validated by real-time quantitative PCR. At last, in order to establish models for predicting prognostic status, the hub genes underwent lasso regression analysis to eliminate collinearity in a cohort of patients receiving ICB therapy. Classification and regression tree (CART) algorithm was a nonparametric, supervised statistical learning technique combining variable values which best discriminated between classes. A machine learning prediction model based on CART analysis was also employed by us to predict the response of patients treated with ICB.

\section{Materials And Methods}

\section{Sample Collection and data reprocessing}

We conducted a systematic search of the electronic databases, including PubMed, GEO profiles, TCGA and EGA datasets. 1601 MIBC transcriptomic profiles from 10 datasets were collected. Details of datasets, including their respective normalizations, were given in Supplementary Table 1. Raw data normalization was carried out in different platforms with default settings[5,11-19]. 13,832 common genes in this study were extracted from the normalized data for six different platforms, and the combat function in the sva package was applied to remove the batch effects of these datasets[20].

Unsupervised immune clusters in MIBC

Immune-related signature genes were selected and listed in Supplementary Table 2. 785 immune genes of 28 immune cell subtypes were obtained from Charoentong et al. [21] Angiogenesis marker genes and antigen MHC I and II presenting machinery expression signature genes involved in antigen-presenting machinery (APM) were obtained from Şenbabaoğlu and Rody et al [22, 23]. Anti-inflammatory and Pro-inflammatory signature genes were obtained from Azizi et al[24]. IFN signature genes were obtained from Rody et al[23]. The unsupervised clustering for 1601 tumor samples based on immune signature genes was performed with hierarchical clustering, Ward linkage, and Euclidean distance.

Single sample Gene Set Enrichment Analysis

Infiltration levels of immune cell subtypes were quantified using the single sample Gene Set Enrichment Analysis (ssGSEA) analysis implementation in R package gsva[25]. ssGSEA analysis also took advantage of the signature 
genes(Supplementary Table 3) to identify immune pathways[15, 22, 23] and metabolic pathways[24] enriched in genes with the highest variability for different clusters[26]. Expression levels for checkpoints were also evaluated.

Somatic mutation analysis

Somatic mutation data were obtained from the publicly available TCGA database via the GDC data portal[27]. From the four subtypes of data files, we selected the "Masked Somatic Mutation" data and processed it based on the VarScan software. We prepared the Mutation Annotation Format (MAF) of somatic variants and implemented the "maftools" [28] R package, which provided multiple analysis modules to perform the visualization process.

Copy-number variation analysis

The TCGA copy-number variation (CNV) data were downloaded from website[29]. Circos plots were performed by the $\mathrm{R}$ package "Rcircos" [30].

DNA methylation analysis

TCGA-HNSC DNA methylation data (Illumina Human Methylation 450k) were downloaded from website[31]. Methylation data were normalized by $\beta$-mixture quantile normalization method (BMIQ) [32]. ChAMP was used to perform quality control, standardization, and calculation of methylation sites and regions [33]. Differentially methylated probes (DMPs)were identified by bumphunter method[34]. We used $95 \%$ quantile as the cut- off value for the candidate regions, which contained at least 7 CpG probe. The heatmap was created in R (v3.4.1) using the pheatmap package on the quantile normalized methylation (beta) values.

Differentially Expressed Genes Screening

The DESeq2 function in the R software package [35] was used to analyze the genetic differences between clusters in TCGA and IMvigor210 cohorts. We screened the DEGs with the adjusted P-value $<0.05$ and |log2(Foldchange) $\mid>1$. Statistical analysis was carried out for each dataset, and the intersecting part of DEGs was identified using the Venn diagram webtool[36].

\section{Gene Ontology Annotations}

Gene Ontology (GO) analysis was performed to show the unique biological significance based on differentially expressed genes. The Cluster Profiler packages [37] in R were applied to analysis and demonstrated GO annotation.

Co-expression Network Construction

WGCNA package of R software was applied to uncover the correlation among genes[38]. The soft-thresholding power $\beta$ was calculated in the construction of each module using the pick Soft Threshold function of WGCNA. The power of $\beta$ was set at 10 to ensure a scale-free network. The minimum number of module genes was set at 30 . The hierarchical clustering dendrogram summarized the Gene modules with different colors. Heat map and topological overlap matrix (TOM) plot were used to visualize the module structure. The relationships between modules and clinic traits were assessed using the correlations between the module eigengene and the clinic trait, allowing the identification of hub modules that were most significantly associated with external traits. For hub modules, the quantitative measurement of module membership was defined as the correlation of the module eigengene and the gene expression profile. Gene significance was defined as the absolute value of the correlation between the genes and the clinic traits. We set the Module Membership $>0.8$ and the GeneSignificance $>0.2$ to select candidate hub genes.

Selection and Validation of Hub Genes related to prognosis

Page $4 / 24$ 
We selected candidate hub genes in TCGA dataset and used the Search Tool for the Retrieval of Interacting Genes (STRING) database[39] to construct PPI network. The confidence score was set at 0.95[40]. Gene network files exported from STRING were input into Cytoscape software. The plug-in cytoHubba of Cytoscape was used for screening out hub genes of the intersecting top 50 genes based on 12 different kinds of Global-based methods. Hub Genes related to prognosis were screened to plot the Kaplan-Meier survival curve in ggplot2 of R software. We also enrolled survival data and expression level of hub genes related to prognosis from an independent cohort IMvigor210.

Clinical samples

17 clinical samples of MIBC and corresponding adjacent tissues were collected from patients treated in Peking Union Medical College Hospital from August 2020 to February 2021. The samples were frozen in liquid nitrogen until mRNA extraction.

Reverse transcription quantitative polymerase chain reaction

Total tissue RNA was extracted using the TriQuick Reagent (Solarbio Life Science). RNA concentration and purity were measured by Nano Drop 2000 system (Thermo Fisher, Carlsbad, USA). Total RNA was reverse transcribed using TranScript $\AA$ First-Strand cDNA synthesis SuperMix (TransGen Biotech). Real-time quantitative polymerase chain reaction (qPCR) was performed using FastStart Essential DNA Green Master (Roche Diagnostics). The PCR reactions were performed on LightCycler® 96 Instrument. SYBR Green was used as the fluorophore. The primer sequences were as follows: LCK: forward, TCTGCACAGCTATGAGCCCT; reverse, GAAGGAGCCGTGAGTGTTCC. PSMB10: forward, TGGCGTCCAAGATGGAGCTA; reverse, GGGGATGCACACCGTAGAG. IL2RB: forward, CTGCTTCACCAACCAGGGTTA; reverse, GGGGTCGTAAGTAAAGTACACCT. CXCR6: forward, GCACACACTGGGAATACTATGC; reverse, CCCTCAGGTATGCGATGGC. IFNG: forward, TCGGTAACTGACTTGAATGTCCA; reverse, TCGCTTCCCTGTTTTAGCTGC. Expression level was calculated relative to control using the $2^{-\triangle \triangle C t}$ method.

Least absolute shrinkage and selection method (LASSO) regression analysis and establishment of prognostic model We used cohort IMvigor210 to establish the prognostic model. The hub genes underwent lasso regression analysis to eliminate collinearity between genes. After performing 1000 10-fold cross-validations, the lambda value with minimized error was selected as the optimum lambda parameter value. We used multivariate Cox proportional hazards regression analysis to find key genes involved in the model. The disease risk score, which was used as predictors of prognostic status, was defined by parameter $\beta$ of the multivariate cox proportional hazards regression analysis and the expression level of each selected gene. The risk score of each patient was calculated, categorized into high or low-risk. The predictive performance of this model at 5-year endpoint was evaluated with a time-dependent receiver operating characteristic (ROC) curve.

CART analysis

We utilized CART analysis to discriminate stable disease (SD) and progressive disease (PD) in IMvigor210 cohort. The optimal combination of variables and possible cutoff values for classification was determined by an exhaustive search of all possibilities by the CART algorithm[41]. The Gini criterion was applied to minimize node impurity after splitting. we also performed 10 -fold cross validation to assess the classified efficiency of the decision tree.

\section{Results}

Molecular immune phenotypes in MIBC patients

Unsupervised clustering of 1601 MIBC patients using immune-related genes was performed. Three main clusters were identified as cluster 1, cluster 2, and cluster 3 (Fig. 1A). ssGSEA scores indicated that Cluster 3 was infiltrated with high levels of innate and adaptive immune cells. Cluster 2 was heterogeneously infiltrated with immune cells while cluster 1 was noninfiltrated. Cluster 3 showed significantly high expression of signatures of CD8 T cells, macrophages, Th1 cells, NK cells, and 
Tregs, followed by cluster 2 and cluster 1 (Fig. 1B). Immune-related pathways, involving interferon (IFN) signaling, TGF-beta signaling, Antigen presenting mechanism (APM), angiogenesis, epithelial-mesenchymal transition (EMT) were mostly enhanced in cluster 3 , followed by cluster 2 and cluster 1 . On the other hand, DNA damage response (DDR) and FGFR3 signaling were mostly enhanced in cluster 3 , followed by cluster 2 and cluster 1 . Cluster 3 was divided into three subclusters, $3 \mathrm{~A}, 3 \mathrm{~B}$ and $3 \mathrm{C}$. cluster 1 was divided into two subclusters, $1 \mathrm{~A}$ and $1 \mathrm{~B}$. Cluster $3 \mathrm{C}$ was the most "inflamed" subcluster with highest level of infiltrated immune cells and activation of immune-related pathways. On the contrary, cluster $1 \mathrm{~A}$ was associated with "non-inflammation" due to the lowest level of infiltrated immune cells and inhibition of immune cells activation. We used RNA-based ESTIMATE algorithm to confirm that cluster $3 \mathrm{C}$ displayed the highest stromal score and immune score, which was the least pure subcluster. The analysis of SSGSEA scores in metabolic pathways showed that hypoxia regulated genes and glucose deprivation related genes were increased in cluster 3 , with a potential to activate immune cells through immunometabolic mechanisms. Cluster 3 also had the best overall survival compared to the other two clusters (Fig. 1C). The expression levels of IFNG, TGF $\beta$, immune checkpoints PD1 and PD-L1 were most enhanced in cluster 3, while the expression level of FGFR3 was most enhanced in cluster 1 (Fig. 1D).

Differences in somatic mutations related to the immune phenotype

After applying unsupervised clustering in TCGA cohort, mutation information of each gene in each sample was exhibited in waterfall plot in different subclusters, as cluster 1A, 1B, 2, 3A, 3B, and 3C (Fig. 2A, 2B, 2C, 2D, 2E, 2F). Various colors with annotations at the bottom represented different mutation types. Detailed information on somatic mutations in different subclusters was shown in supplementary Fig. 1. There was a gradual increase of RB1 mutations and a gradual decease of TTN mutations from cluster 1A to 3C. We also identified a lower frequency of KDM6A mutations in cluster 3C compared with other subclusters. EP300 mutations was relatively less frequent in $1 \mathrm{~A}$ than other subclusters.

DEGs between cluster $3 \mathrm{C}$ and cluster $1 \mathrm{~A}$

After applying unsupervised clustering in TCGA and IMvigor210 cohorts, DEGs between cluster 3C and cluster 1A were analyzed. 3790 significantly up-regulated DEGs and 4794 down-regulated DEGs were identified in TCGA dataset. In IMvigor210 cohort, 2950 up-regulated genes and 1067 down-regulated genes are identified. The intersection includes 2043 significantly up-regulated and 662 down-regulated genes (Fig. 3A). The volcano plots of TCGA cohort and IMvigor 210 cohort were shown (Fig. 3B). The GO analysis of upregulated DEGs in cluster $3 \mathrm{C}$ was enriched in immune cell activation involving $T$ cell activation, leukocyte activation, phagocytosis, and leukocyte migration (Fig. 3C). KEGG analysis showed that the downregulated genes in cluster $3 \mathrm{C}$ were enriched in various metabolic pathways involving amino acid metabolism and fatty acid degradation (Fig. 3D).

Difference in genomic CNVs and methylation in DEGs between cluster 3C and cluster 1A

We then performed an integrated analysis of Multi-omics data on TCGA cohorts. To evaluate whether copy number variations (CNVs) affect transcription of affected genes, analysis of genomic alteration of DEGs indicated regions of copynumber gains (chromosomes 1,2,3,4,10,12,17,19,21) or loss (chromosomes 2,6,9,10,11,17) as characteristic features of cluster 1A compare with cluster 3C in the TCGA cohort (Fig. 4A). 161 upregulated DEGs in cluster 3C were coded by regions with a higher frequency for copy-number deletions in cluster $1 \mathrm{~A}$, while 37 upregulated DEGs in cluster $1 \mathrm{~A}$ were encoded by regions with more frequent gains in the cluster (Fig. 4B). GO analysis of 161 upregulated DEGs with copy-number gains in cluster $3 \mathrm{C}$ was enriched in cell adhesion, inflammatory response, and integral component of plasma membrane (Fig. 4C). Global methylation data available for the TCGA cohort were analyzed. In total, differentially methylated probes of 349 genes were identified comparing cluster $1 \mathrm{~A}$ and cluster $3 \mathrm{C}$. Probes with significantly higher beta values in cluster $3 \mathrm{C}$ were located in the proximal promoter of 25 DEGs with higher expression in cluster $1 \mathrm{~A}$, while Probes with significantly higher beta values in cluster $1 \mathrm{~A}$ were located in the proximal promoter of 16 DEGs with higher expression in cluster 3C (Fig. 4D). 4 genes were identified in both CNV and methylation analysis, of which 2 DEGs, ARHGAP25 and MKX, were upregulated in cluster 3C and 2 DEGs, MRAP2 and SNAP91, were upregulated in cluster 1A (Fig. 4E). 
WGCNA analysis

The intersected 2705 DEGs were used to perform subsequent WGCNA analysis. The power of $\beta$ was set at 10 to ensure a scale-free network (Fig. 5C). Gene modules were calculated. The gray module identified the genes that can't be classified to other modules. 12 gene modules were identified by the hierarchical clustering dendrogram (Fig. 5A). The relationships between modules and clinic traits were assessed. Many modules are correlated with survival time. The correlation coefficients of the red, yellow, blue, and pink modules were greatest, at 0.28 (0.001), 0.28 (0.001), 0.25 (0.005), and 0.23 (0.009), respectively (Fig. 5B). MM and GS value in the upper quartile of genes in the module were considered as key genes of this module (The MM and GS cut-off of these modules were 0.8 and 0.2 , respectively). The gene distribution of red, yellow, blue and pink modules was shown (Fig. 5D). A total of 415 hub genes were investigated from 4 modules, which were analyzed using matascape for pathway and process enrichment analysis. GO analysis indicate these genes are closely related to cytokine-mediated signaling, regulation of cell activation, regulation of cytokine production and leukocyte differentiation (Fig. 5E).

Identification and validation of hub genes

Protein-protein interactions (PPI) were applied to identify 8 genes as hub genes (LCK, HLA-E, IRF1, IL2RB, IFNG, CCL13, CXCR6, PSMB10) (Fig. 6A). To validate our finding of hub genes, we examined TCGA and IMvigor210 cohorts. The KM curves for genes with $\mathrm{P}<0.05$ in TCGA cohort were shown in supplementary Fig. 2. IFNG, CXCR6, IL2RB, LCK, and PSMB10 were found to be closely related to prognosis in both cohorts (Fig. 7A, 7B, 7C, 7D, and 7E). We constructed an immune infiltration interaction network based on the 5 hub genes using the STRING dataset. The results were imported into Cytoscape for visualization (Fig. 6B). Real-time qPCR results indicated that the expression levels of LCK, PSMB10, CXCR6, IL2RB, and IFNG were significantly lower in cancer tissues than in normal bladder tissues (Fig. 8A).

In order to establish a model for predicting prognostic status, 5 genes were used to perform a lasso regression, with 10-fold cross-validation with 1000 repeats. $\lambda$ value with the smallest Partial Likelyhood Deviance was shown in 2 of the 5 genes had coefficients that were not zero. A prognostic score model involving LCK and PSMB10 was established.

Risk Score $=(-0.1973 \times$ LCK $)+(-0.0091 \times$ PSMB10 $)$

By predicting survival of patients at 5 years, the areas under the curve (AUC) of the ROC curves generated from the risk-based prediction model in the test data were 0.652 (Fig. 7F). The test data were obtained from IMvigor210 cohorts, consisting of patients receiving ICB therapy.

The decision tree illustrated decision rules, among all the 5 hub genes that were entered in the analysis, 4 were selected by the program for the classification tree (Fig. 8B). The four parameters were LCK, IFNG, PSMB10, and CXCR6. LCK was the most essential determining factor, which was the first-level split of two initial branches of the classification tree. The mean accuracy, sensitivity, and specificity for predicting SD/PD were $70.1 \%, 70.0 \%$ and $71.7 \%$.

\section{Discussion}

A novel molecular classifier based on immune-related genes was established by us. Unsupervised clustering divided our cohorts into three clusters, Cluster 1, 2, and 3. Cluster 3 was termed as most "inflammed" while cluster 1 was identified as "non-inflammation" according to the difference in infiltrated immune cells and immune-related signaling. Cluster 3C showed the highest expression level of CD8 T cells, which are primed and activated to cytotoxic T lymphocytes (CTLs). CTLs are the key immune cells for killing malignant cells. CD8 T cells priming requires IFN-y secreted by Th1 cells and NK cells and TNF-a secreted by Macrophages[42]. The expression levels of Th1, NK cells, and Macrophages were also the highest in cluster 3. ssGESA score of immune-related signaling showed high levels of IFN signaling and TGF- $\beta$ signaling in cluster 3 . IFN upregulates APM, which displayed the highest level in cluster 3. Upregulation of APM could present more antigen and increase the number of CD8 + T cells. IFN-y also plays an important role in the regulation of PD-L1 expression[43]. Tumors 
with high IFN- $\gamma$ levels are more likely to respond to anti-PD-1 therapy[44]. Moreover, IFN- $\gamma$ was suggested to have a protective response limiting the process of bladder cancer[45]. TGF- $\beta$ is the effector molecule of macrophages and it's associated with stromal activation, angiogenesis and epithelial-mesenchymal transition (EMT), which were induced in cluster 3, with protumorigenic effects[46]. Besides, cluster 3 displayed lowest expression in genes involved in DNA damage response (DDR), which increased tumor mutation burden, likely inducing immune cells activation and increasing response to ICB therapy[47]. Cluster 1 showed the highest level of FGFR3 signaling. FGFR is associated with a lack of T-cell inflammation[48], leading to a "non-inflammation" phenotype. Metabolic regulation is essential for immune cells activation. Hypoxia regulated genes were significantly increased in cluster 3 , which might lead to increased activation of fatty acid catabolism and CD $8+T$ cells activation[49]. Glucose deprivation related genes were also induced in cluster 3, increasing PD-1 expression on CD8 + T cells. Metabolically, Glycolysis is reduced while fatty acid metabolism is induced in immune cells[50]. However, direct evidence on metabolism regulating immune cells in tumor microenvironment needs further studies.

The analysis of somatic mutation indicated a higher frequency of RB1 mutations and a lower frequency of KDM6A mutations in cluster 3C. A recent review concluded that RB1 activation related to antigen presentation and interferon response, enhancing the mobilization of immune cells into the tumor microenvironment[51]. RB1 mutations tended to gather in basal-squamous subtype, while KDM6A mutations were more common in luminal subtype[5], which were consistent with the distributions of the clusters (Fig. 1A). EP300 mutations were least frequent in cluster 1A and were found with higher tumor mutation burden and improved antitumor immunity in bladder cancer[52], probably related to the poor prognosis of cluster 1A. 4 DEGs were selected based on genomic CNVs and DNA methylation analyses. ARHGAP25 is a novel Rac GTPase-activating protein upregulated in cluster 3C. Researches indicated that ARHGAP25 negatively regulated phagocytosis of immune cells and affected trans-endothelial migration of neutrophilic granulocytes[53, 54]. ARHGAP25 was found to inhibit colorectal cancer metastasis through Wnt/ $\beta$-catenin pathway[55]. A similar mechanism was also demonstrated in lung cancer[56]. The role of ARHGAP25 in bladder cancer require further investigations. SNAP91 upregulated in cluster $1 \mathrm{~A}$ was found to relate to vesicle-mediated transport and clathrin-mediated endocytosis. Researches indicated that SNAP91 was decreased in glioblastoma and esophageal cancer[57, 58], while SNAP91 was overexpressed in metastatic prostate cancer[59]. The role of SNAP91 in bladder cancer requires more studies. Investigations on MRAP2 and MKX in tumors are currently rare, which might be a hint for future researches. The analysis of somatic mutation, genomic CNVs and DNA methylation in our study didn't show direct relation to the 5 hub genes selected by WGCNA analysis. The molecular mechanisms regulating the expression of the hub genes require further studies in the future.

WGCNA plus PPI analysis identified 5 hub genes, IFNG, CXCR6, IL2RB, LCK, and PSMB10, which were correlated with survival time. Real-time qPCR results also confirmed that the expression levels of the 5 hub genes were significantly higher in bladder tissues adjacent to tumor. In our study, higher expression of CXCR6 was correlated with higher survival rate. However, CXCR6 has been shown to predict poor prognosis in gastric cancer, breast cancer, prostate cancer and bladder cancer[60-63]. On the contrary, CXCR6 was also found to be necessary for promoting NKT and CD4 T cells to remove senescent hepatocytes to prevent hepatocarcinogenesis[64], which might indicate a similar mechanism in MIBC. Interleukin-2 (IL-2) was one of the first FDA-approved immunotherapy drugs for melanoma and renal cell cancer. IL-2 receptor (IL-2R) complex is important to control immune response[65, 66]. There are three IL-2R subunits: IL-2Ra, IL-2Rß, and IL-2 $\gamma$. The application of IL-2 treatment was limited because it induced proliferation of Tregs through IL-2 Ra, which is preferentially expressed on Tregs[67].

Increased Tregs are correlated with worse prognosis[68, 69]. On the contrary, CD8 T cells and NK cells express IL-2R $\beta$ and IL$2 \gamma$, which lack IL-2 Ra. The activation of CD8 T cells and NK cells contribute to a better prognosis. Strategies targeting IL-2R $\beta$ have shown effective tumor control through activating CD8 T cells and NK cells[70, 71]. Lymphocyte-specific protein tyrosine kinase (LCK) was essential for TCR signaling, associated with Th1, Th2, and Th17 cell differentiation[72]. As a result, LCK has become a druggable target to improve the efficacy of chimeric antigen receptors and to potentiate T-cell response in immunotherapy[73]. Another study indicated that LCK was associated with bladder cancer prognosis, which was consistent with our results[74]. Proteasome subunit beta type-10 (PSMB10) is one of the immunoproteasome subunits. Interferon- $y$ induces the expression of the catalytic immunoproteasomes[75]. PSMB10 plays a pivotal role in antigen generation/processing, which is crucial for cytotoxic T lymphocytes to eliminate cancer cells[76, 77]. Upregulation of

Page $8 / 24$ 
immunoproteasomes in breast cancer shown an abundance of tumor infiltrating lymphocytes and a good prognosis[78]. A similar mechanism might be discovered in bladder cancer patients.

\section{Conclusions}

A prognostic score model involving LCK and PSMB10 was established to predict patients undergoing ICB therapy. In addition, the decision tree selected 4 genes including LCK, PSMB10, IFNG, and CXCR6 by the program. qPCR results indicated that expression levels all of the hub genes were markedly higher in bladder tissues compared to bladder cancers. Hopefully, the hub genes selected by us and machine learning prediction model could help predict the MIBC patients' response to ICB therapy. However, further Researches on MIBC patients receiving ICB therapy should be carried out to validate our prediction model. Besides, the mechanisms regulating selected hub genes require further studies. Exploring molecular mechanisms regulating hub genes may also help us find potential targets to treat MIBC.

\section{List Of Abbreviations}

MIBC Muscle-invasive bladder cancer

AUC areas under the curve

ICB Immune checkpoint blockade

WGCNA Weighted Gene Co-expression Network Analyses

CART Classification and regression tree

qPCR quantitative polymerase chain reaction

NMIBC non-muscle-invasive bladder cancer

PD-L1 programmed death ligand-1

DEGs differentially expressed genes

APM antigen presenting machinery

CNV copy-number variation

GO Gene Ontology

KEGG The Kyoto Encyclopedia of Genes and Genomes

GSEA Gene Set Enrichment Analysis

$\mathrm{ROC}$ receiver operating characteristic

SD stable disease

PD progressive disease

IFN interferon

EMT epithelial-mesenchymal transition

DDR DNA damage response

Page 9/24 
PPI Protein-protein interactions

CTL cytotoxic T lymphocytes

IL-2 Interleukin-2

IL-2R IL-2 receptor

LCK Lymphocyte-specific protein tyrosine kinase

PSMB10 Proteasome subunit beta type-10

\section{Declarations}

\section{Ethic approval and consent to participate}

The Institutional Review Board (IRB) in Peking Union Medical College Hospital has reviewed our protocol and has determined that 1) the applicant(s) are qualified to conduct the proposed study; 2) the design of the study is rationale and scientifically sound; 3) human subject protection is adequate. In compliance with the Declaration of Helsinki, the IRB approve our protocol.

\section{Consent for publication}

Not applicable.

\section{Availability of data and materials}

Most of the research data are available at GEO profiles, TCGA and EGA datasets. Other data generated or analyzed during this study are included in this published article and its additional files.

\section{Competing interests}

The authors declare that they have no competing interests.

\section{Funding}

None.

\section{Author contributions}

Pei-Heng Li and Zhi-xin Chen designed the study and collected the data. Zhi-Xin Chen analyzed the data. Pei-Heng Li interpreted the results. Dong Wang, Zhi Zheng, and Zhi-Gang Ji corrected the results and interpretation of data. All authors read and approved the final manuscript. 
None.

\section{References}

[1] F. Bray, J. Ferlay, I. Soerjomataram, R.L. Siegel, L.A. Torre, A. Jemal, Global cancer statistics 2018: GLOBOCAN estimates of incidence and mortality worldwide for 36 cancers in 185 countries, CA: a cancer journal for clinicians, 68 (2018) 394-424.

[2] J. Alfred Witjes, T. Lebret, E.M. Comperat, N.C. Cowan, M. De Santis, H.M. Bruins, V. Hernandez, E.L. Espinos, J. Dunn, M. Rouanne, Y. Neuzillet, E. Veskimae, A.G. van der Heijden, G. Gakis, M.J. Ribal, Updated 2016 EAU Guidelines on Muscleinvasive and Metastatic Bladder Cancer, Eur Urol, 71 (2017) 462-475.

[3] T.W. Flaig, P.E. Spiess, N. Agarwal, R. Bangs, S.A. Boorjian, M.K. Buyyounouski, T.M. Downs, J.A. Efstathiou, T. Friedlander, R.E. Greenberg, K.A. Guru, N. Hahn, H.W. Herr, C. Hoimes, B.A. Inman, M. Jimbo, A.K. Kader, S.M. Lele, J.J. Meeks, J. Michalski, J.S. Montgomery, L.C. Pagliaro, S.K. Pal, A. Patterson, D.P. Petrylak, E.R. Plimack, K.S. Pohar, M.P. Porter, M.A. Preston, W.J. Sexton, A.O. Siefker-Radtke, J. Tward, G. Wile, A. Johnson-Chilla, M.A. Dwyer, L.A. Gurski, NCCN Guidelines Insights: Bladder Cancer, Version 5.2018, J Natl Compr Canc Netw, 16 (2018) 1041-1053.

[4] V. Dadhania, M. Zhang, L. Zhang, J. Bondaruk, T. Majewski, A. Siefker-Radtke, C.C. Guo, C. Dinney, D.E. Cogdell, S. Zhang, S. Lee, J.G. Lee, J.N. Weinstein, K. Baggerly, D. McConkey, B. Czerniak, Meta-Analysis of the Luminal and Basal Subtypes of Bladder Cancer and the Identification of Signature Immunohistochemical Markers for Clinical Use, EBioMedicine, 12 (2016) 105-117.

[5] A. Kamoun, A. de Reynies, Y. Allory, G. Sjodahl, A.G. Robertson, R. Seiler, K.A. Hoadley, C.S. Groeneveld, H. Al-Ahmadie, W. Choi, M.A.A. Castro, J. Fontugne, P. Eriksson, Q. Mo, J. Kardos, A. Zlotta, A. Hartmann, C.P. Dinney, J. Bellmunt, T. Powles, N. Malats, K.S. Chan, W.Y. Kim, D.J. McConkey, P.C. Black, L. Dyrskjot, M. Hoglund, S.P. Lerner, F.X. Real, F. Radvanyi, G. Bladder Cancer Molecular Taxonomy, A Consensus Molecular Classification of Muscle-invasive Bladder Cancer, Eur Urol, 77 (2020) 420-433.

[6] N. van Dijk, S.A. Funt, C.U. Blank, T. Powles, J.E. Rosenberg, M.S. van der Heijden, The Cancer Immunogram as a Framework for Personalized Immunotherapy in Urothelial Cancer, Eur Urol, 75 (2019) 435-444.

[7] J. Jung, H. Zeng, T. Horng, Metabolism as a guiding force for immunity, Nat Cell Biol, 21 (2019) 85-93.

[8] L.A. O'Neill, R.J. Kishton, J. Rathmell, A guide to immunometabolism for immunologists, Nat Rev Immunol, 16 (2016) 553565.

[9] B. Zhang, S. Horvath, A general framework for weighted gene co-expression network analysis, Statistical applications in genetics and molecular biology, 4 (2005) Article17.

[10] T. Davoli, H. Uno, E.C. Wooten, S.J. Elledge, Tumor aneuploidy correlates with markers of immune evasion and with reduced response to immunotherapy, Science (New York, N.Y.), 355 (2017).

[11] D. Lindgren, A. Frigyesi, S. Gudjonsson, G. Sjodahl, C. Hallden, G. Chebil, S. Veerla, T. Ryden, W. Mansson, F. Liedberg, M. Hoglund, Combined gene expression and genomic profiling define two intrinsic molecular subtypes of urothelial carcinoma and gene signatures for molecular grading and outcome, Cancer Res, 70 (2010) 3463-3472.

[12] M. Riester, J.M. Taylor, A. Feifer, T. Koppie, J.E. Rosenberg, R.J. Downey, B.H. Bochner, F. Michor, Combination of a novel gene expression signature with a clinical nomogram improves the prediction of survival in high-risk bladder cancer, Clin Cancer Res, 18 (2012) 1323-1333. 
[13] W. Choi, S. Porten, S. Kim, D. Willis, E.R. Plimack, J. Hoffman-Censits, B. Roth, T. Cheng, M. Tran, I.L. Lee, J. Melquist, J. Bondaruk, T. Majewski, S. Zhang, S. Pretzsch, K. Baggerly, A. Siefker-Radtke, B. Czerniak, C.P. Dinney, D.J. McConkey, Identification of distinct basal and luminal subtypes of muscle-invasive bladder cancer with different sensitivities to frontline chemotherapy, Cancer Cell, 25 (2014) 152-165.

[14] R. Seiler, H.A.D. Ashab, N. Erho, B.W.G. van Rhijn, B. Winters, J. Douglas, K.E. Van Kessel, E.E. Fransen van de Putte, M. Sommerlad, N.Q. Wang, V. Choeurng, E.A. Gibb, B. Palmer-Aronsten, L.L. Lam, C. Buerki, E. Davicioni, G. Sjodahl, J. Kardos, K.A. Hoadley, S.P. Lerner, D.J. McConkey, W. Choi, W.Y. Kim, B. Kiss, G.N. Thalmann, T. Todenhofer, S.J. Crabb, S. North, E.C. Zwarthoff, J.L. Boormans, J. Wright, M. Dall'Era, M.S. van der Heijden, P.C. Black, Impact of Molecular Subtypes in Muscleinvasive Bladder Cancer on Predicting Response and Survival after Neoadjuvant Chemotherapy, Eur Urol, 72 (2017) 544-554.

[15] S. Mariathasan, S.J. Turley, D. Nickles, A. Castiglioni, K. Yuen, Y. Wang, E.E. Kadel, III, H. Koeppen, J.L. Astarita, R. Cubas, S. Jhunjhunwala, R. Banchereau, Y. Yang, Y. Guan, C. Chalouni, J. Ziai, Y. Senbabaoglu, S. Santoro, D. Sheinson, J. Hung, J.M. Giltnane, A.A. Pierce, K. Mesh, S. Lianoglou, J. Riegler, R.A.D. Carano, P. Eriksson, M. Hoglund, L. Somarriba, D.L. Halligan, M.S. van der Heijden, Y. Loriot, J.E. Rosenberg, L. Fong, I. Mellman, D.S. Chen, M. Green, C. Derleth, G.D. Fine, P.S. Hegde, R. Bourgon, T. Powles, TGFbeta attenuates tumour response to PD-L1 blockade by contributing to exclusion of T cells, Nature, 554 (2018) 544-548.

[16] J.J. de Jong, Y. Liu, A.G. Robertson, R. Seiler, C.S. Groeneveld, M.S. van der Heijden, J.L. Wright, J. Douglas, M. Dall'Era, S.J. Crabb, B.W.G. van Rhijn, K.E.M. van Kessel, E. Davicioni, M.A.A. Castro, Y. Lotan, E.C. Zwarthoff, P.C. Black, J.L. Boormans, E.A. Gibb, Long non-coding RNAs identify a subset of luminal muscle-invasive bladder cancer patients with favorable prognosis, Genome Med, 11 (2019) 60.

[17] J.A. Efstathiou, K.W. Mouw, E.A. Gibb, Y. Liu, C.L. Wu, M.R. Drumm, J.B. da Costa, M. du Plessis, N.Q. Wang, E. Davicioni, F.Y. Feng, R. Seiler, P.C. Black, W.U. Shipley, D.T. Miyamoto, Impact of Immune and Stromal Infiltration on Outcomes Following Bladder-Sparing Trimodality Therapy for Muscle-Invasive Bladder Cancer, Eur Urol, 76 (2019) 59-68.

[18] R. Seiler, E.A. Gibb, N.Q. Wang, H.Z. Oo, H.M. Lam, K.E. van Kessel, C.S. Voskuilen, B. Winters, N. Erho, M.M. Takhar, J. Douglas, F. Vakar-Lopez, S.J. Crabb, B.W.G. van Rhijn, E.E. Fransen van de Putte, E.C. Zwarthoff, G.N. Thalmann, E. Davicioni, J.L. Boormans, M. Dall'Era, M.S. van der Heijden, J.L. Wright, P.C. Black, Divergent Biological Response to Neoadjuvant Chemotherapy in Muscle-invasive Bladder Cancer, Clin Cancer Res, 25 (2019) 5082-5093.

[19] B.N. Song, S.K. Kim, J.Y. Mun, Y.D. Choi, S.H. Leem, I.S. Chu, Identification of an immunotherapy-responsive molecular subtype of bladder cancer, EBioMedicine, 50 (2019) 238-245.

[20] L. Xia, X. Su, J. Shen, Q. Meng, J. Yan, C. Zhang, Y. Chen, H. Wang, M. Xu, ANLN functions as a key candidate gene in cervical cancer as determined by integrated bioinformatic analysis, Cancer Manag Res, 10 (2018) 663-670.

[21] P. Charoentong, F. Finotello, M. Angelova, C. Mayer, M. Efremova, D. Rieder, H. Hackl, Z. Trajanoski, Pan-cancer Immunogenomic Analyses Reveal Genotype-Immunophenotype Relationships and Predictors of Response to Checkpoint Blockade, Cell Rep, 18 (2017) 248-262.

[22] Y. Şenbabaoğlu, R.S. Gejman, A.G. Winer, M. Liu, E.M. Van Allen, G. de Velasco, D. Miao, I. Ostrovnaya, E. Drill, A. Luna, N. Weinhold, W. Lee, B.J. Manley, D.N. Khalil, S.D. Kaffenberger, Y. Chen, L. Danilova, M.H. Voss, J.A. Coleman, P. Russo, V.E. Reuter, T.A. Chan, E.H. Cheng, D.A. Scheinberg, M.O. Li, T.K. Choueiri, J.J. Hsieh, C. Sander, A.A. Hakimi, Tumor immune microenvironment characterization in clear cell renal cell carcinoma identifies prognostic and immunotherapeutically relevant messenger RNA signatures, Genome Biol, 17 (2016) 231.

[23] A. Rody, T. Karn, C. Liedtke, L. Pusztai, E. Ruckhaeberle, L. Hanker, R. Gaetje, C. Solbach, A. Ahr, D. Metzler, M. Schmidt, V. Müller, U. Holtrich, M. Kaufmann, A clinically relevant gene signature in triple negative and basal-like breast cancer, Breast Cancer Res, 13 (2011) R97.

Page $12 / 24$ 
[24] E. Azizi, A.J. Carr, G. Plitas, A.E. Cornish, C. Konopacki, S. Prabhakaran, J. Nainys, K. Wu, V. Kiseliovas, M. Setty, K. Choi, R.M. Fromme, P. Dao, P.T. McKenney, R.C. Wasti, K. Kadaveru, L. Mazutis, A.Y. Rudensky, D. Pe'er, Single-Cell Map of Diverse Immune Phenotypes in the Breast Tumor Microenvironment, Cell, 174 (2018) 1293-1308.e1236.

[25] S. Hänzelmann, R. Castelo, J. Guinney, GSVA: gene set variation analysis for microarray and RNA-seq data, BMC Bioinformatics, 14 (2013) 7.

[26] D.A. Barbie, P. Tamayo, J.S. Boehm, S.Y. Kim, S.E. Moody, I.F. Dunn, A.C. Schinzel, P. Sandy, E. Meylan, C. Scholl, S. Frohling, E.M. Chan, M.L. Sos, K. Michel, C. Mermel, S.J. Silver, B.A. Weir, J.H. Reiling, Q. Sheng, P.B. Gupta, R.C. Wadlow, H. Le, S. Hoersch, B.S. Wittner, S. Ramaswamy, D.M. Livingston, D.M. Sabatini, M. Meyerson, R.K. Thomas, E.S. Lander, J.P. Mesirov, D.E. Root, D.G. Gilliland, T. Jacks, W.C. Hahn, Systematic RNA interference reveals that oncogenic KRAS-driven cancers require TBK1, Nature, 462 (2009) 108-112.

[27] https:// portal.gdc.cancer.gov/

[28] A. Mayakonda, D.C. Lin, Y. Assenov, C. Plass, H.P. Koeffler, Maftools: efficient and comprehensive analysis of somatic variants in cancer, Genome Res, 28 (2018) 1747-1756.

[29]https://tcga.xenahubs.net/download/TCGA.BLCA.sampleMap/Gistic2_CopyNumber_Gistic2_all_thresholded.by_genes.gz

[30] M. Ayers, J. Lunceford, M. Nebozhyn, E. Murphy, A. Loboda, D.R. Kaufman, A. Albright, J.D. Cheng, S.P. Kang, V. Shankaran, S.A. Piha-Paul, J. Yearley, T.Y. Seiwert, A. Ribas, T.K. McClanahan, IFN-gamma-related mRNA profile predicts clinical response to PD-1 blockade, J Clin Invest, 127 (2017) 2930-2940.

[31] https://tcga.xenahubs.net/download/TCGA.BLCA.sampleMap/HumanMethylation450.gz

[32] A.E. Teschendorff, F. Marabita, M. Lechner, T. Bartlett, J. Tegner, D. Gomez-Cabrero, S. Beck, A beta-mixture quantile normalization method for correcting probe design bias in Illumina Infinium 450 k DNA methylation data, Bioinformatics, 29 (2013) 189-196.

[33] Y. Tian, T.J. Morris, A.P. Webster, Z. Yang, S. Beck, A. Feber, A.E. Teschendorff, ChAMP: updated methylation analysis pipeline for Illumina BeadChips, Bioinformatics, 33 (2017) 3982-3984.

[34] L.A. Williams, L. Mills, A.J. Hooten, E. Langer, M. Roesler, A.L. Frazier, M. Krailo, H.H. Nelson, J. Bestrashniy, J.F. Amatruda, J.N. Poynter, Differences in DNA methylation profiles by histologic subtype of paediatric germ cell tumours: a report from the Children's Oncology Group, Br J Cancer, 119 (2018) 864-872.

[35] J. Reimand, R. Isserlin, V. Voisin, M. Kucera, C. Tannus-Lopes, A. Rostamianfar, L. Wadi, M. Meyer, J. Wong, C. Xu, D. Merico, G.D. Bader, Pathway enrichment analysis and visualization of omics data using g:Profiler, GSEA, Cytoscape and EnrichmentMap, Nat Protoc, 14 (2019) 482-517.

[36] http://bioinformatics.psb.ugent.be/webtools/Venn/

[37] G. Yu, L.G. Wang, Y. Han, Q.Y. He, clusterProfiler: an R package for comparing biological themes among gene clusters, OMICS, 16 (2012) 284-287.

[38] Z. Liu, M. Li, X. Fang, L. Shen, W. Yao, Z. Fang, J. Chen, X. Feng, Hu, Z. Zeng, C. Lin, J. Weng, Y. Lai, G. Yi, Identification of surrogate prognostic biomarkers for allergic asthma in nasal epithelial brushing samples by WGCNA, J Cell Biochem, 120 (2019) 5137-5150.

[39] https:// string-db.org/ 
[40] D. Szklarczyk, A.L. Gable, D. Lyon, A. Junge, S. Wyder, J. Huerta-Cepas, M. Simonovic, N.T. Doncheva, J.H. Morris, P. Bork, L.J. Jensen, C.V. Mering, STRING v11: protein-protein association networks with increased coverage, supporting functional discovery in genome-wide experimental datasets, Nucleic Acids Res, 47 (2019) D607-D613.

[41] S.C. Lemon, J. Roy, M.A. Clark, P.D. Friedmann, W. Rakowski, Classification and regression tree analysis in public health: methodological review and comparison with logistic regression, Annals of behavioral medicine : a publication of the Society of Behavioral Medicine, 26 (2003) 172-181.

[42] B. Farhood, M. Najafi, K. Mortezaee, CD8(+) cytotoxic T lymphocytes in cancer immunotherapy: A review, J Cell Physiol, 234 (2019) 8509-8521.

[43] A. Garcia-Diaz, D.S. Shin, B.H. Moreno, J. Saco, H. Escuin-Ordinas, G.A. Rodriguez, J.M. Zaretsky, L. Sun, W. Hugo, X. Wang, G. Parisi, C.P. Saus, D.Y. Torrejon, T.G. Graeber, B. Comin-Anduix, S. Hu-Lieskovan, R. Damoiseaux, R.S. Lo, A. Ribas, Interferon Receptor Signaling Pathways Regulating PD-L1 and PD-L2 Expression, Cell reports, 19 (2017) 1189-1201.

[44] S. Maleki Vareki, High and low mutational burden tumors versus immunologically hot and cold tumors and response to immune checkpoint inhibitors, Journal for immunotherapy of cancer, 6 (2018) 157.

[45] E. Ahlen Bergman, C.A. Hartana, M. Johansson, L.B. Linton, S. Berglund, M. Hyllienmark, C. Lundgren, B. Holmstrom, K. Palmqvist, J. Hansson, F. Alamdari, Y. Huge, F. Aljabery, K. Riklund, M.E. Winerdal, D. Krantz, A.A. Zirakzadeh, P. Marits, L.K. Sjoholm, A. Sherif, O. Winqvist, Increased CD4(+) T cell lineage commitment determined by CpG methylation correlates with better prognosis in urinary bladder cancer patients, Clin Epigenetics, 10 (2018) 102.

[46] R.J. Akhurst, A. Hata, Targeting the TGF $\beta$ signalling pathway in disease, Nature reviews. Drug discovery, 11 (2012) $790-$ 811.

[47] M.Y. Teo, K. Seier, I. Ostrovnaya, A.M. Regazzi, B.E. Kania, M.M. Moran, C.K. Cipolla, M.J. Bluth, J. Chaim, H. Al-Ahmadie, A. Snyder, M.I. Carlo, D.B. Solit, M.F. Berger, S. Funt, J.D. Wolchok, G. Iyer, D.F. Bajorin, M.K. Callahan, J.E. Rosenberg, Alterations in DNA Damage Response and Repair Genes as Potential Marker of Clinical Benefit From PD-1/PD-L1 Blockade in Advanced Urothelial Cancers, Journal of clinical oncology : official journal of the American Society of Clinical Oncology, 36 (2018) 1685-1694.

[48] R.F. Sweis, S. Spranger, R. Bao, G.P. Paner, W.M. Stadler, G. Steinberg, T.F. Gajewski, Molecular Drivers of the Non-T-cellInflamed Tumor Microenvironment in Urothelial Bladder Cancer, Cancer immunology research, 4 (2016) 563-568.

[49] N.R. Maimela, S. Liu, Y. Zhang, Fates of CD8+ T cells in Tumor Microenvironment, Comput Struct Biotechnol J, 17 (2019) $1-13$.

[50] C.H. Chang, J.D. Curtis, L.B. Maggi, Jr., B. Faubert, A.V. Villarino, D. O'Sullivan, S.C. Huang, G.J. van der Windt, J. Blagih, J. Qiu, J.D. Weber, E.J. Pearce, R.G. Jones, E.L. Pearce, Posttranscriptional control of T cell effector function by aerobic glycolysis, Cell, 153 (2013) 1239-1251.

[51] E.S. Knudsen, S.C. Pruitt, P.A. Hershberger, A.K. Witkiewicz, D.W. Goodrich, Cell Cycle and Beyond: Exploiting New RB1 Controlled Mechanisms for Cancer Therapy, Trends in Cancer, 5 (2019) 308-324.

[52] G. Zhu, L. Pei, Y. Li, X. Gou, EP300 mutation is associated with tumor mutation burden and promotes antitumor immunity in bladder cancer patients, Aging, 12 (2020) 2132-2141.

[53] R. Csepanyi-Komi, E. Wisniewski, B. Bartos, P. Levai, T. Nemeth, B. Balazs, A.R. Kurz, S. Bierschenk, M. Sperandio, E. Ligeti, Rac GTPase Activating Protein ARHGAP25 Regulates Leukocyte Transendothelial Migration in Mice, Journal of immunology (Baltimore, Md. : 1950), 197 (2016) 2807-2815. 
[54] R. Csepanyi-Komi, G. Sirokmany, M. Geiszt, E. Ligeti, ARHGAP25, a novel Rac GTPase-activating protein, regulates phagocytosis in human neutrophilic granulocytes, Blood, 119 (2012) 573-582.

[55] L. Tao, Y. Zhu, Y. Gu, J. Zheng, J. Yang, ARHGAP25: A negative regulator of colorectal cancer (CRC) metastasis via the Wnt/beta-catenin pathway, Eur J Pharmacol, 858 (2019) 172476.

[56] K. Xu, B. Liu, Y. Ma, The tumor suppressive roles of ARHGAP25 in lung cancer cells, Onco Targets Ther, 12 (2019) 66996710.

[57] Y.F. Gao, X.Y. Mao, T. Zhu, C.X. Mao, Z.X. Liu, Z.B. Wang, L. Li, X. Li, J.Y. Yin, W. Zhang, H.H. Zhou, Z.Q. Liu, COL3A1 and SNAP91: novel glioblastoma markers with diagnostic and prognostic value, Oncotarget, 7 (2016) 70494-70503.

[58] F. Pan, M. Chen, X.Y. Song, J.D. Yang, MicroRNA-301b and its target gene synaptosome-associated protein 91 as important modulators in esophageal cancer: functional experiments, Anticancer Drugs, 31 (2020) 411-422.

[59] Y. Sun, G. Chen, J. He, Z.G. Huang, S.H. Li, Y.P. Yang, L.Y. Zhong, S.F. Ji, Y. Huang, X.H. Chen, M.L. He, H. Wu, Clinical significance and potential molecular mechanism of miRNA-222-3p in metastatic prostate cancer, Bioengineered, 12 (2021) 325-340.

[60] J.T. Lee, S.D. Lee, J.Z. Lee, M.K. Chung, H.K. Ha, Expression analysis and clinical significance of CXCL16/CXCR6 in patients with bladder cancer, Oncol Lett, 5 (2013) 229-235.

[61] G. Xiao, X. Wang, J. Wang, L. Zu, G. Cheng, M. Hao, X. Sun, Y. Xue, J. Lu, J. Wang, CXCL16/CXCR6 chemokine signaling mediates breast cancer progression by pERK1/2-dependent mechanisms, Oncotarget, 6 (2015) 14165-14178.

[62] R. Singh, N. Kapur, H. Mir, N. Singh, J.W. Lillard, Jr., S. Singh, CXCR6-CXCL16 axis promotes prostate cancer by mediating cytoskeleton rearrangement via Ezrin activation and av $\beta 3$ integrin clustering, Oncotarget, 7 (2016) 7343-7353.

[63] J.J. Jin, F.X. Dai, Z.W. Long, H. Cai, X.W. Liu, Y. Zhou, Q. Hong, Q.Z. Dong, Y.N. Wang, H. Huang, CXCR6 predicts poor prognosis in gastric cancer and promotes tumor metastasis through epithelial-mesenchymal transition, Oncology reports, 37 (2017) 3279-3286.

[64] J.C. Mossanen, M. Kohlhepp, A. Wehr, O. Krenkel, A. Liepelt, A.A. Roeth, D. Mockel, F. Heymann, T. Lammers, N. Gassler, J. Hermann, J. Jankowski, U.P. Neumann, T. Luedde, C. Trautwein, F. Tacke, CXCR6 Inhibits Hepatocarcinogenesis by Promoting Natural Killer T- and CD4(+) T-Cell-Dependent Control of Senescence, Gastroenterology, 156 (2019) 1877-1889 e1874.

[65] S.A. Rosenberg, J.C. Yang, D.E. White, S.M. Steinberg, Durability of complete responses in patients with metastatic cancer treated with high-dose interleukin-2: identification of the antigens mediating response, Annals of surgery, 228 (1998) 307-319.

[66] S.A. Rosenberg, IL-2: the first effective immunotherapy for human cancer, Journal of immunology (Baltimore, Md. : 1950), 192 (2014) 5451-5458.

[67] M. Ahmadzadeh, S.A. Rosenberg, IL-2 administration increases CD4+ CD25(hi) Foxp3+ regulatory T cells in cancer patients, Blood, 107 (2006) 2409-2414.

[68] S.D. Barth, J.J. Schulze, T. Kühn, E. Raschke, A. Hüsing, T. Johnson, R. Kaaks, S. Olek, Treg-Mediated Immune Tolerance and the Risk of Solid Cancers: Findings From EPIC-Heidelberg, Journal of the National Cancer Institute, 107 (2015).

[69] H. Imai, M. Saio, K. Nonaka, T. Suwa, N. Umemura, G.F. Ouyang, J. Nakagawa, H. Tomita, S. Osada, Y. Sugiyama, Y. Adachi, T. Takami, Depletion of CD4+CD25+ regulatory T cells enhances interleukin-2-induced antitumor immunity in a mouse model of colon adenocarcinoma, Cancer science, 98 (2007) 416-423. 
[70] Z. Sun, Z. Ren, K. Yang, Z. Liu, S. Cao, S. Deng, L. Xu, Y. Liang, J. Guo, Y. Bian, H. Xu, J. Shi, F. Wang, Y.X. Fu, H. Peng, A next-generation tumor-targeting IL-2 preferentially promotes tumor-infiltrating CD8(+) T-cell response and effective tumor control, Nat Commun, 10 (2019) 3874.

[71] Y. Jounaidi, J.F. Cotten, K.W. Miller, S.A. Forman, Tethering IL2 to Its Receptor IL2Rbeta Enhances Antitumor Activity and Expansion of Natural Killer NK92 Cells, Cancer Res, 77 (2017) 5938-5951.

[72] K. Nika, C. Soldani, M. Salek, W. Paster, A. Gray, R. Etzensperger, L. Fugger, P. Polzella, V. Cerundolo, O. Dushek, T. Höfer, A. Viola, O. Acuto, Constitutively active Lck kinase in T cells drives antigen receptor signal transduction, Immunity, 32 (2010) 766-777.

[73] U. Bommhardt, B. Schraven, L. Simeoni, Beyond TCR Signaling: Emerging Functions of Lck in Cancer and Immunotherapy, Int J Mol Sci, 20 (2019).

[74] Y. Zhang, D.H. Ou, D.W. Zhuang, Z.F. Zheng, M.E. Lin, In silico analysis of the immune microenvironment in bladder cancer, BMC Cancer, 20 (2020) 265.

[75] A.L. Goldberg, M. Gaczynska, E. Grant, M. Michalek, K.L. Rock, Functions of the proteasome in antigen presentation, Cold Spring Harbor symposia on quantitative biology, 60 (1995) 479-490.

[76] D. de Verteuil, T.L. Muratore-Schroeder, D.P. Granados, M.H. Fortier, M.P. Hardy, A. Bramoullé, E. Caron, K. Vincent, S. Mader, S. Lemieux, P. Thibault, C. Perreault, Deletion of immunoproteasome subunits imprints on the transcriptome and has a broad impact on peptides presented by major histocompatibility complex I molecules, Molecular \& cellular proteomics : MCP, 9 (2010) 2034-2047.

[77] N. Aptsiauri, T. Cabrera, A. Garcia-Lora, M.A. Lopez-Nevot, F. Ruiz-Cabello, F. Garrido, MHC class I antigens and immune surveillance in transformed cells, International review of cytology, 256 (2007) 139-189.

[78] A. Rouette, A. Trofimov, D. Haberl, G. Boucher, V.P. Lavallee, G. D'Angelo, J. Hebert, G. Sauvageau, S. Lemieux, C. Perreault, Expression of immunoproteasome genes is regulated by cell-intrinsic and -extrinsic factors in human cancers, Sci Rep, 6 (2016) 34019.

\section{Figures}




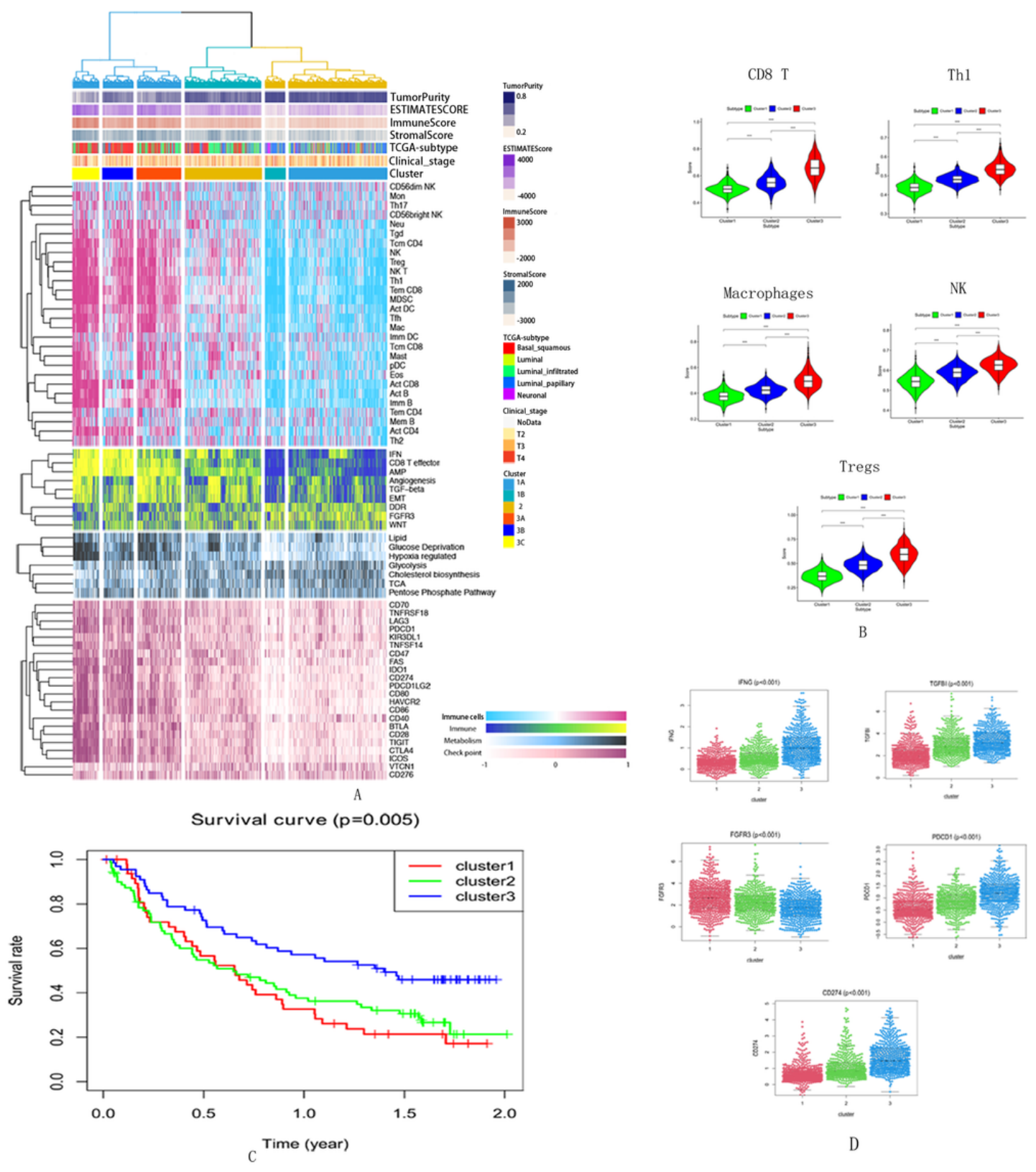

Figure 1

Molecular immune subtypes in MIBC patients. A Unsupervised clustering of 1601 MIBC patients using ssGSEA scores from immune cell types and immune-related pathways. Hierarchical clustering was performed with Euclidean distance and Ward Linkage. Three main clusters were identified as cluster 1, cluster 2 and cluster 3 . Of the three clusters, subcluster $3 \mathrm{C}$ was the most "inflamed" while subcluster 1A was "non-inflammation". ssGSEA analysis of metabolic pathways in different clusters was also shown. B The level of infiltrated immune cells, CD8 T cells, Th1 cells, macrophages, NK cells, and Tregs in the three clusters. Cluster 3 was infiltrated with highest level of innate and adaptive immune cells, followed by cluster 2 and cluster 1. C Survival curves of the three clusters. Cluster 3 was correlated with the best overall survival, followed by cluster 2 and cluster $1(p=0.005)$. D The expression levels of IFNG, TGF $\beta$, FGFR3, immune checkpoints PD1 and PD-L1 in the three clusters. 


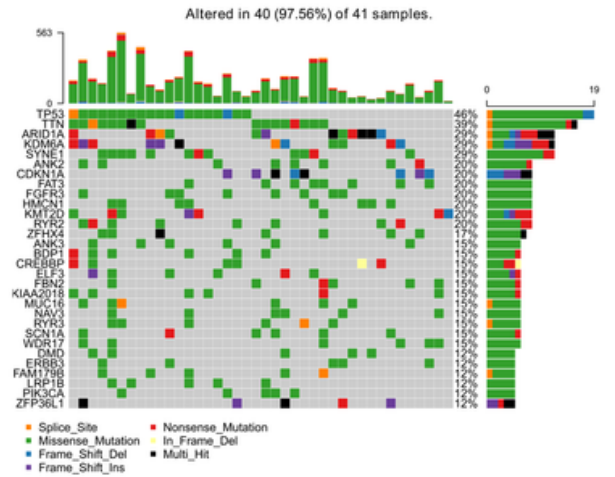

A

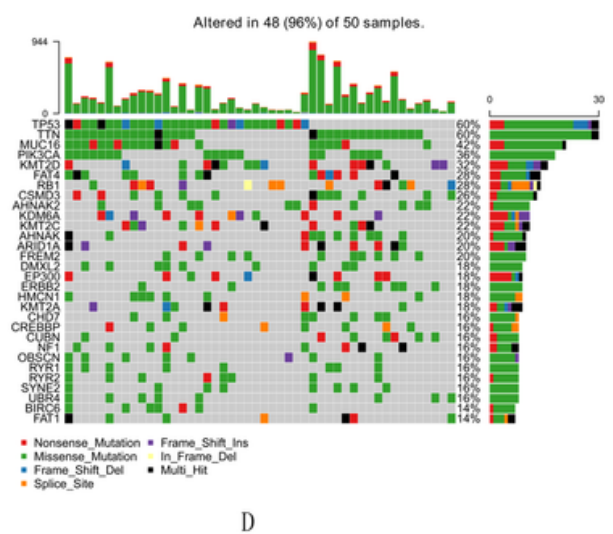

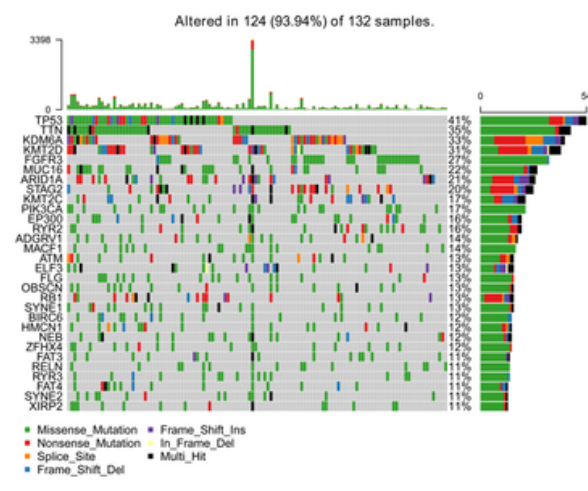

B
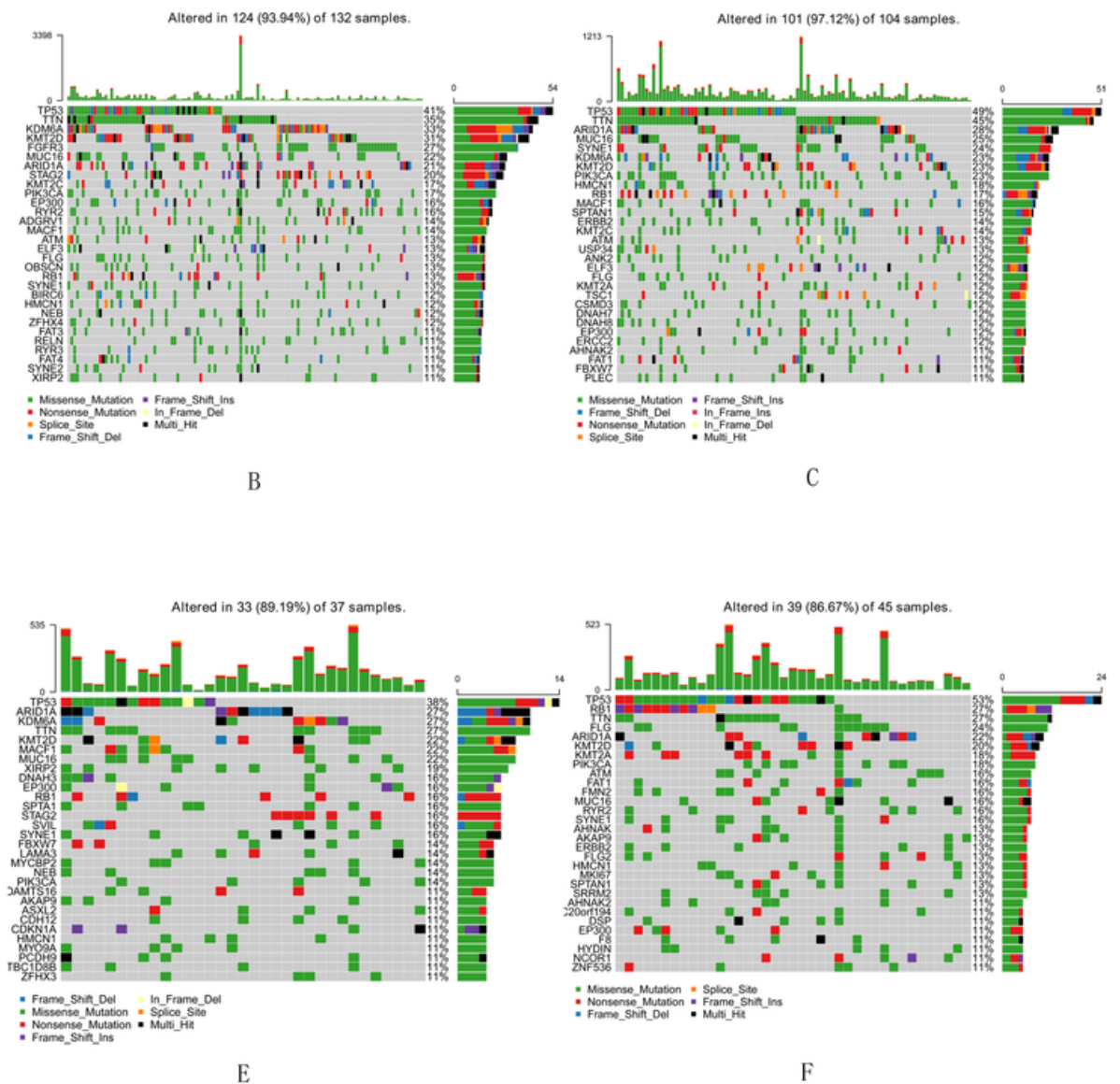

Figure 2

Landscape of somatic mutation profiles in TCGA cohorts. Mutation information of each gene in each sample of six subclusters was shown in the waterfall plot. A, B, C, D, E, F represented cluster 1A, 1B, 2, 3A, 3B, 3C, respectively. Various colors with annotations at the bottom represented the different mutation types. The barplot above the legend exhibited the mutation burden. 

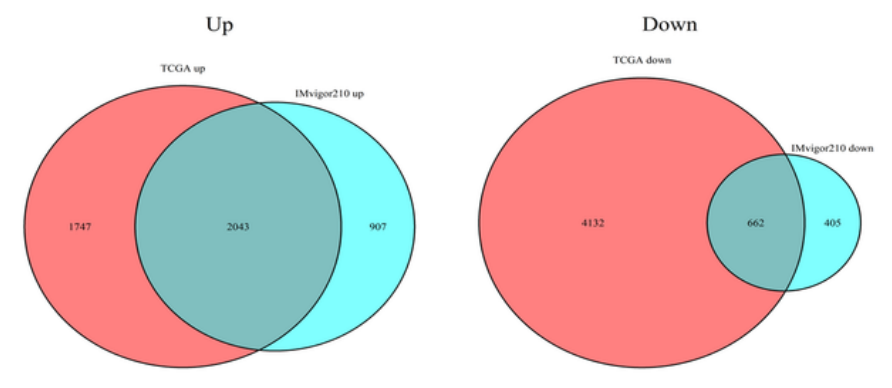

A
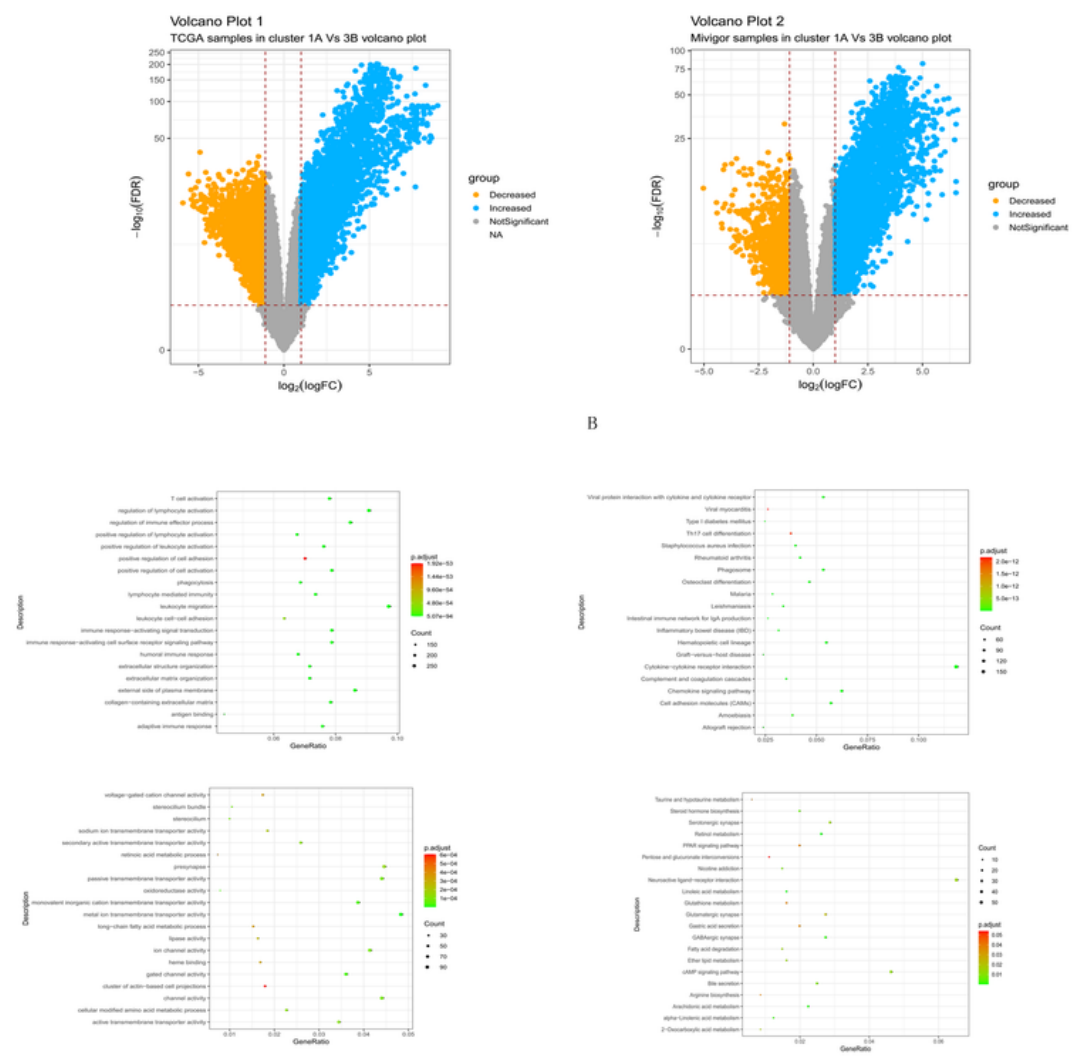

c

D

\section{Figure 3}

DEGs between cluster 1A and cluster 3C. A Venn diagram of upregulated and downregulated DEGs of the TCGA cohort and IMvigor210 cohort. B Volcano plots of DEGs in TCGA and IMvigor 210 cohort. C The GO analysis of upregulated and downregulated DEGs in cluster 3C. D KEGG analysis of upregulated and downregulated genes in cluster 3C. 

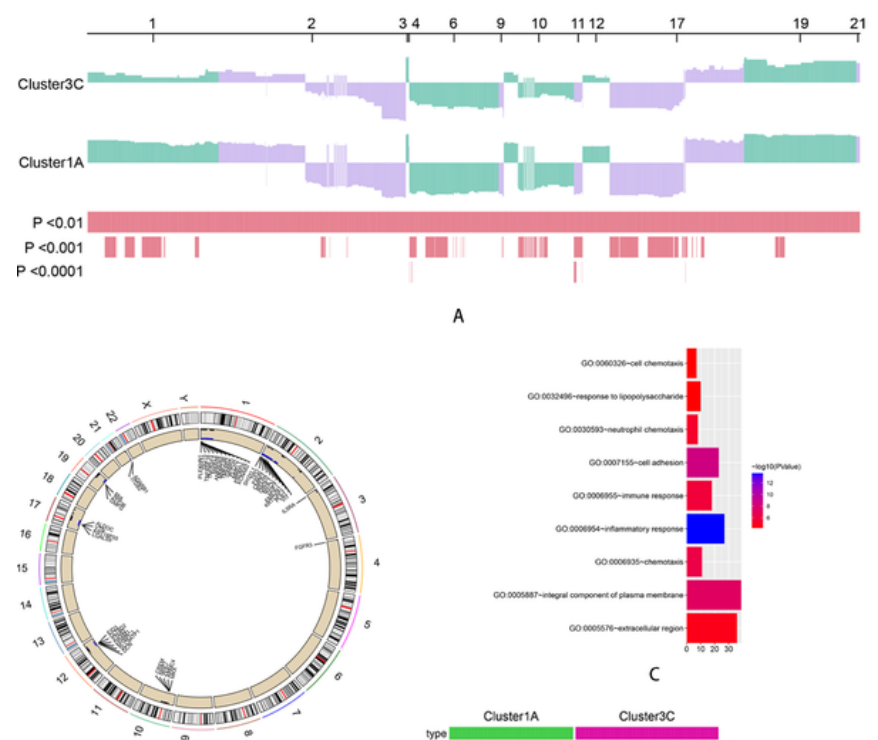

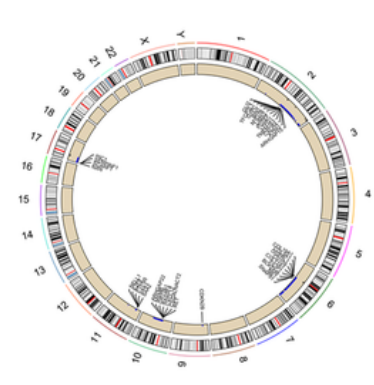

B

Up

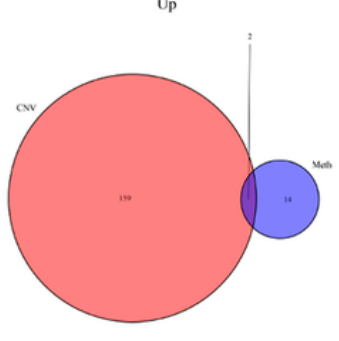

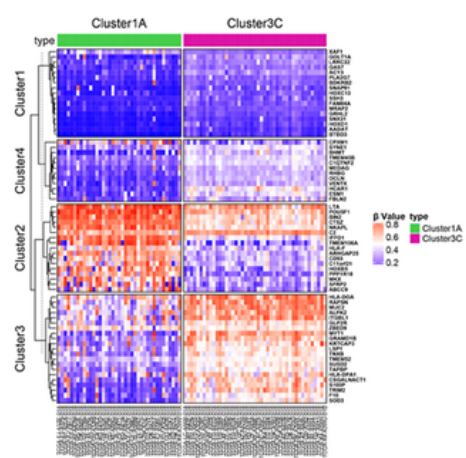

D

Down

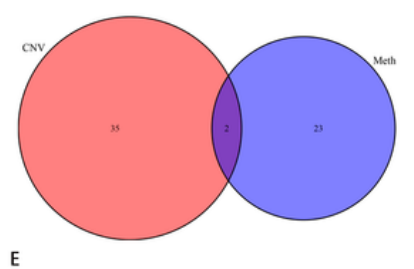

\section{Figure 4}

Differences in CNVs and DNA methylation among cluster 1A and cluster 3C. A CNV plot shows relative frequency of copynumber gains and deletions among DEGs between cluster $1 \mathrm{~A}$ and 3C. $161 \mathrm{DEG}$ were upregulated in cluster 3C in regions with a higher frequency for copy-number deletions in cluster 1A, while $37 \mathrm{DEG}$ were upregulated in cluster $1 \mathrm{~A}$ encoded by regions with more frequent gains in the cluster. B Circos plot display chromosomal positions with statistically significant differences in gains or losses between cluster 1A and cluster 3C. C GO analysis of 161 upregulated DEGs with copy-number gains in cluster 3C. D 349 differentially methylated probes were extracted comparing cluster 1A and cluster 3C. Heatmap shows 41 differentially methylated probes annotated for promoters of DEGs among cluster 1A versus cluster 3C. E Venn diagram demonstrating intersection of DEGs identified in both CNV and methylation analysis. 


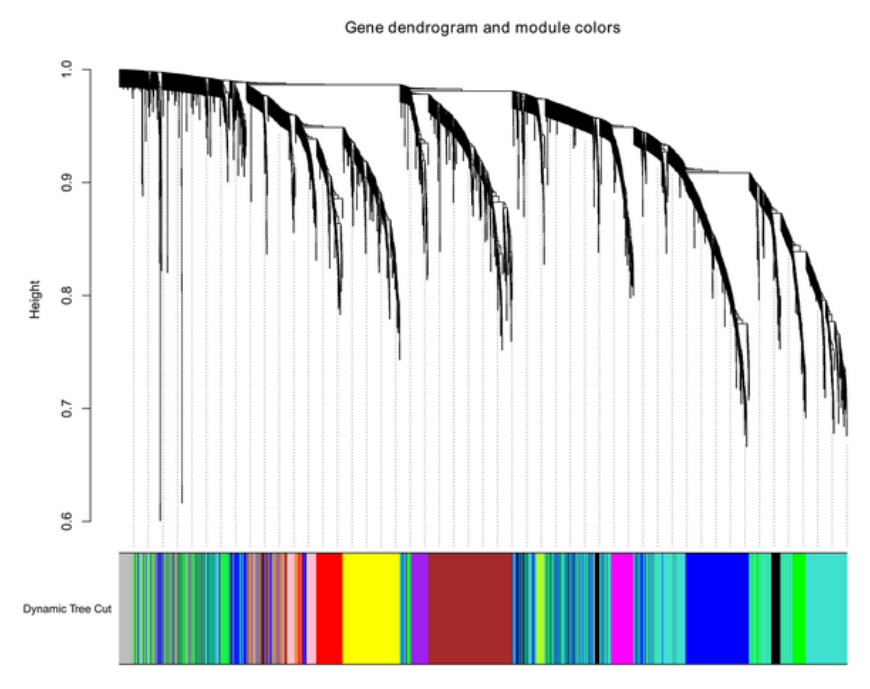

A
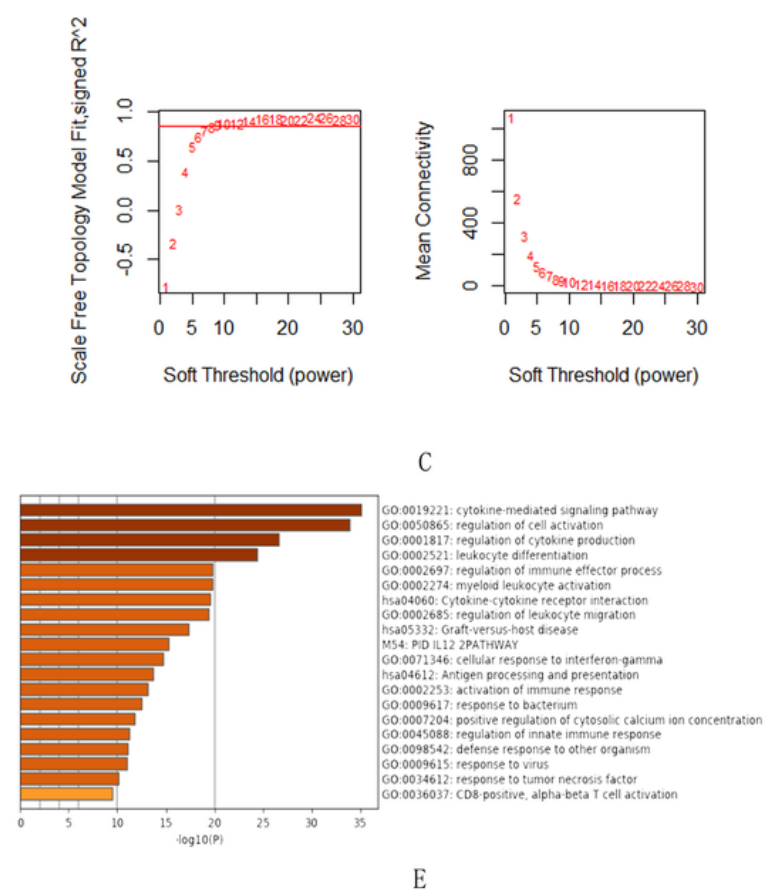
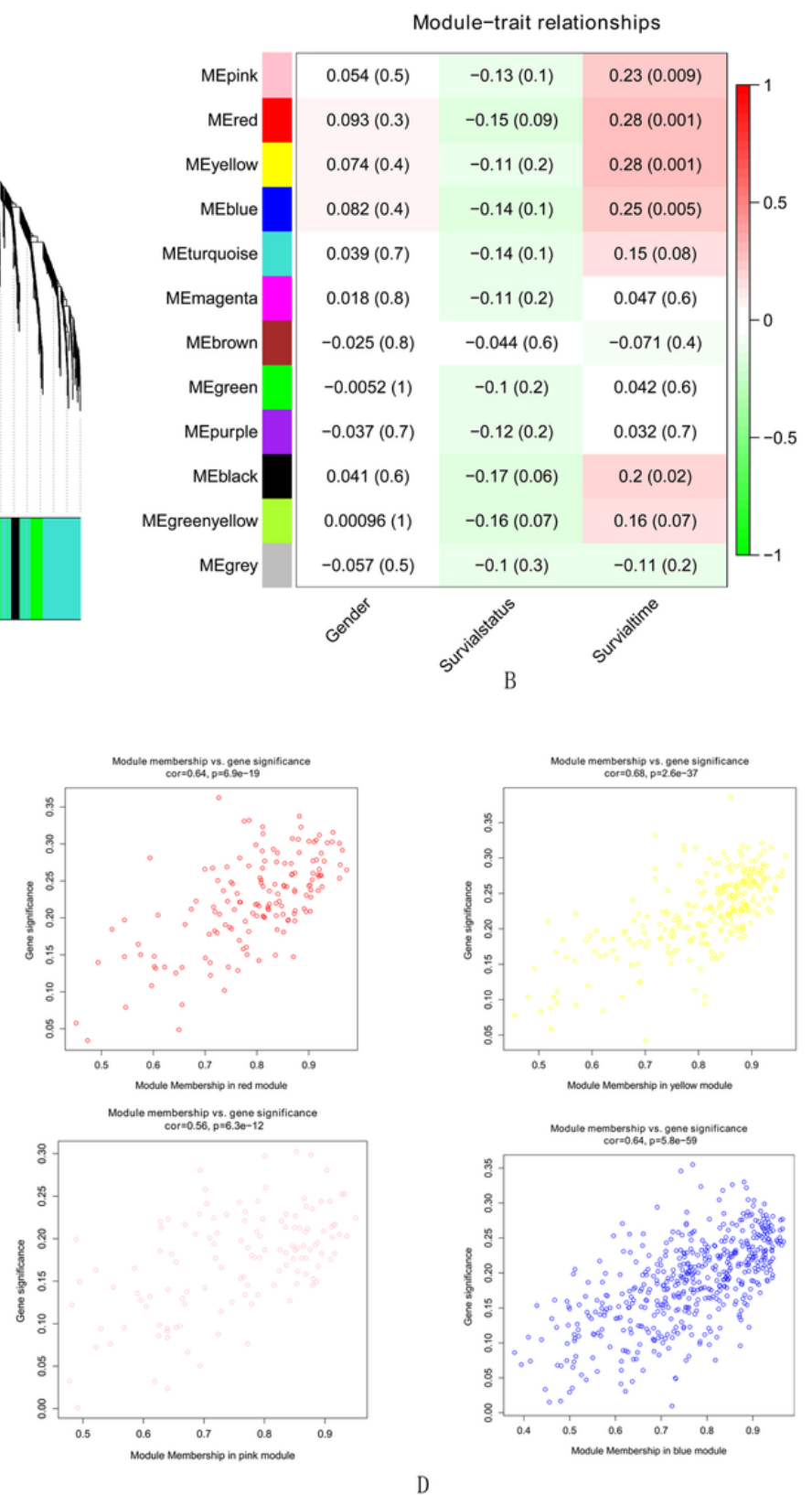

\section{Figure 5}

WGCNA analysis of DEGs between cluster 1A and cluster 3C. A Clustering dendrograms showing 12 modules that contain a group of highly connected genes. Each designated color represents a certain gene module. B Heatmap shows correlations of module epigengenes with survival time. C Determination of soft threshold in WGCNA algorithm. The power of $\beta$ was set at 10 to ensure a scale-free network. D Gene correlation scatter plots of the red, yellow, pink, and blue module. E GO analysis of the four modules. 


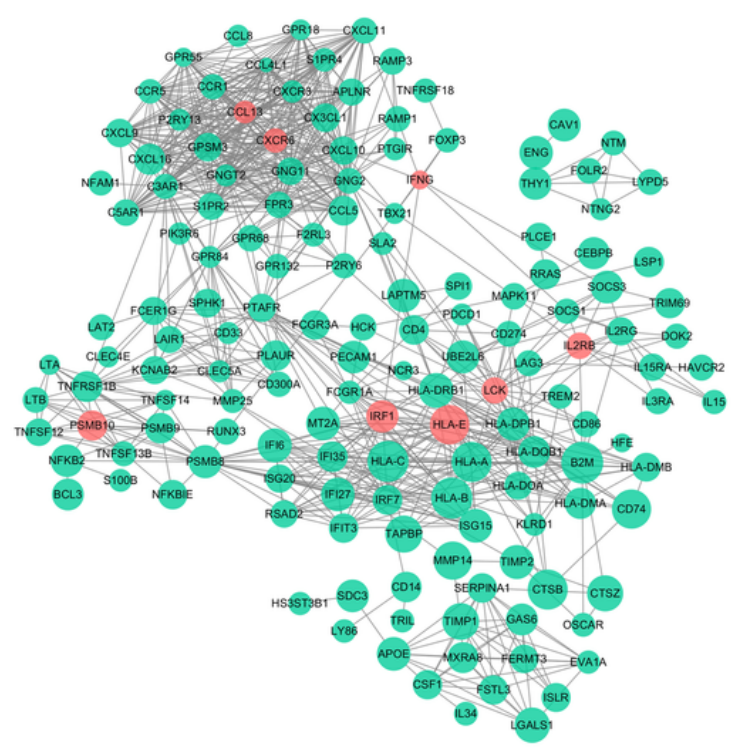

A

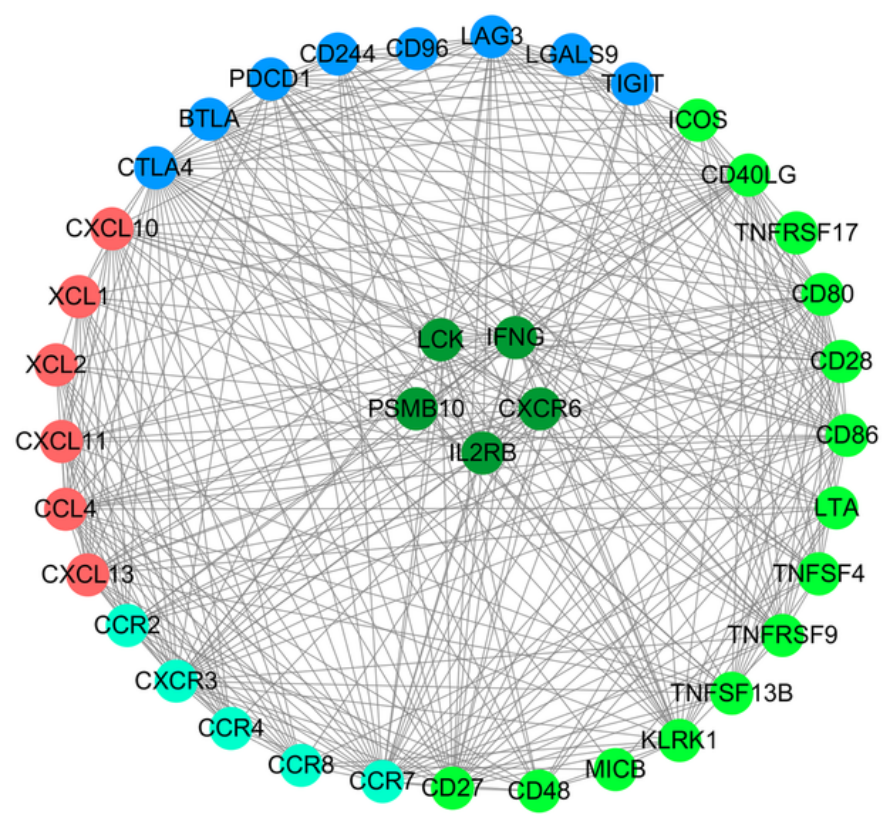

B

Figure 6

PPI. A The visualization of modules with hub genes. The thicker line represents higher connection strengths. B PPI map of the 5 hub genes. 

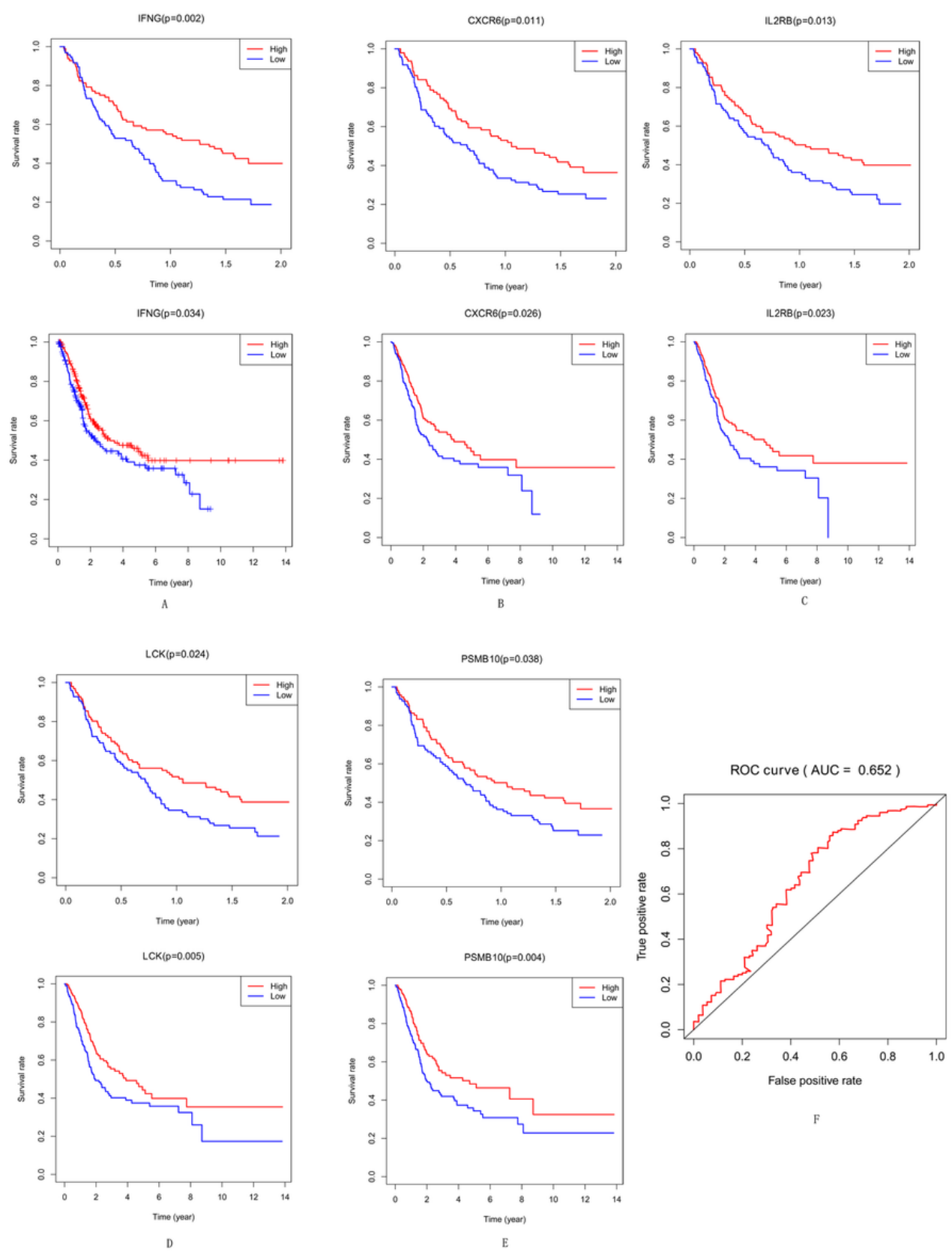

Figure 7

Selection and validation of hub genes related to prognosis. Survival curves for testing hub genes, IFNG, CXCR6, IL2RB, LCK, and PSMB10 in TCGA cohort and IMvigor210 cohort were shown in A, B, C, D, and E. ROC curve for the model representing 5year prediction was shown in $\mathrm{F}$. 

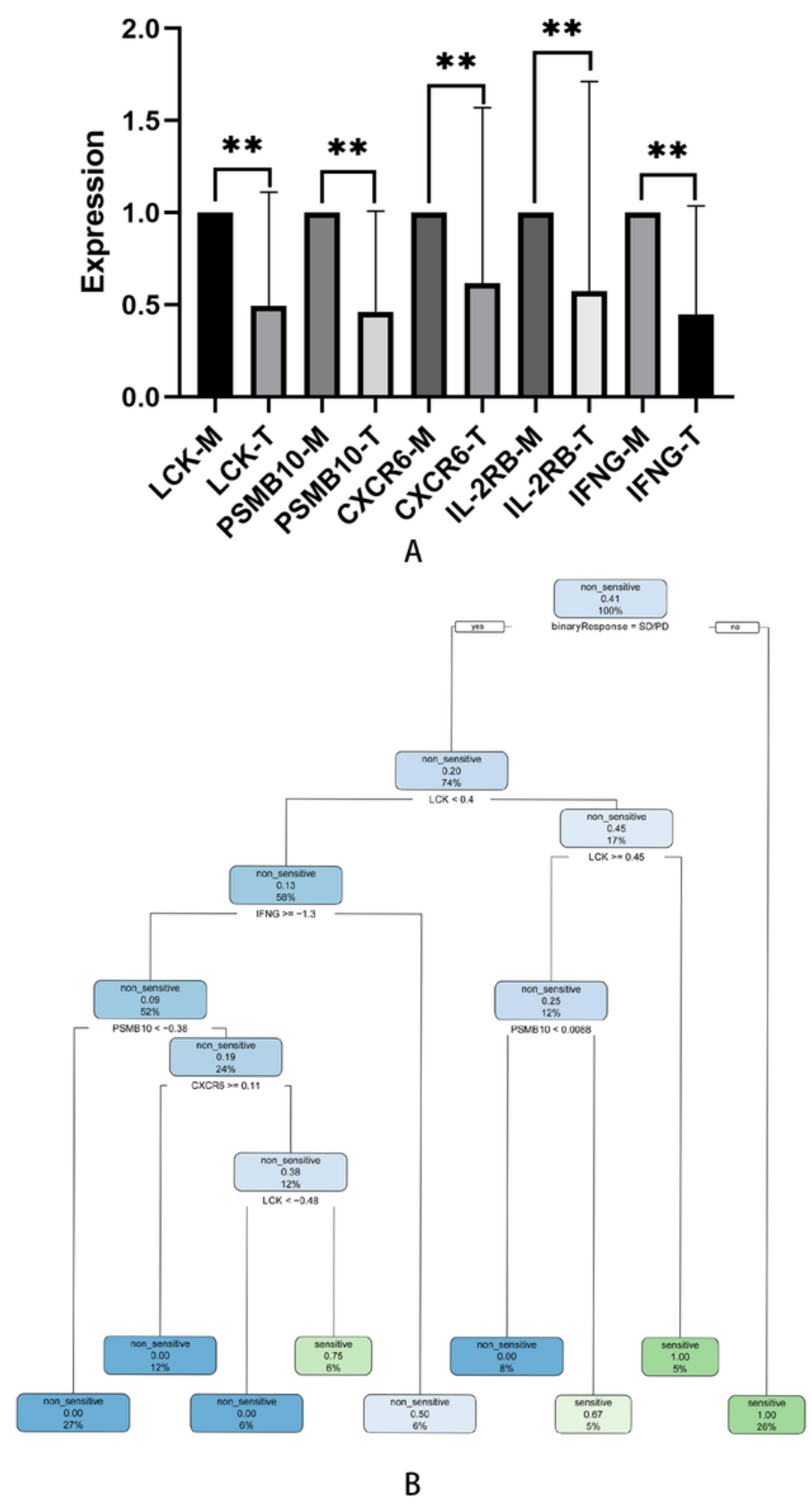

Figure 8

The expression levels of hub genes and decision tree. A Real-time qPCR results of the expression levels of hub genes in bladder cancer tissues (LCK-T, PSMB10-T, CXCR6-T, IL-2RB-T, IFNG-T) and adjacent tissues (LCK-M, PSMB10-M, CXCR6-M, IL2RB-M, IFNG-M). B Decision tree calculated by CART to classify SD and PD patients receiving ICB therapy. 